\title{
Outpatient Submaxillectomy Using Active Drains. Safety of the Procedure and Patient Satisfaction
}

\author{
José-Antonio García-de $\operatorname{Marcos}^{1}$ (D) Alvaro del Amo-Fernández de Velasco ${ }^{1}$. \\ Javier Fernández Alba-Luengo ${ }^{1}$
}

Received: 26 October 2021 / Accepted: 12 November 2021

(C) The Association of Oral and Maxillofacial Surgeons of India 2021

\begin{abstract}
Surgical interventions on an outpatient basis are defined as those procedures in which the patient is discharged the same day of the surgery after monitoring the subject during the immediate postoperative period. Submaxillectomies have conventionally been performed on an inpatient basis, and hospital stay was determined by the drain removal. This study aims to establish both safety and patient satisfaction following outpatient submaxillectomies using active drains. Fifteen patients between March 2016 and March 2020 underwent submaxillectomies using active drains in an outpatient basis. No patient had to be readmitted, and there were no complications that could be associated with the outpatient basis of this procedure, showing the safety of this surgical regimen. Patient satisfaction degree was high, with $86.7 \%$ of the patients declaring low or no pain as well as appropriate hospital stay. Most of the patients stated that they would accept the surgery in an outpatient basis once again. The performance of an outpatient submandibular gland removal is a safe and well-accepted procedure by the patient.
\end{abstract}

Keywords Submaxillectomy · Outpatient surgery · Submandibular gland resection · Drain

José-Antonio García-de Marcos pepio2@hotmail.com

1 Department of Oral and Maxillofacial Surgery, Guadalajara University Hospital, C/Donantes de Sangre, s/n, Guadalajara, Spain

\section{Introduction}

In the current health and economical context, policies aim to increase the efficiency of the resources without impairment of the care and satisfaction of the patients [1,2].

Outpatient surgery, also known as day surgery, is the one that after its performance and immediate postoperative monitoring, the patient is discharged home from hospital the same day of the procedure, irrespective the type of anesthesia applied [1,3]. Day surgeries are divided into major outpatient surgery (MOS), including moderate complexity surgeries under locoregional or general anesthesia, and minor outpatient surgery, reserved for less complex procedures under local anesthesia. The main aim of this technique is to reduce costs following some directions: no decrease in health care and medical results and acceptance by the patient [3].

Submandibular gland removal is a frequent procedure in head and neck surgery, commonly performed as an isolated technique for tumoral or lithiasic pathology of the submaxillary gland. Gland sialoliths can be treated either by removal of the stone or by the complete removal of the gland including the stones, depending on their location. Hilar or intraglandular sialoliths proximal to the posterior border of the mylohyoid muscle often require the resection of the gland [4].

The use of percutaneous drains following a submaxillectomy is considered a standard procedure in the postoperative period of this surgery [5-7]. These drains collapse the surgical site, preventing dead spaces that could eventually lead to blood, saliva or serum formation, and therefore improving wound healing [2].

Submandibular gland removal has traditionally been performed in an inpatient basis, being the time for drain removal the main factor for hospital stay. These drains are 
kept in place for at least $24 \mathrm{~h}$ and removed depending on the amount of drainage and surgeon preferences [1].

There is a growing interest in taking into account the patient's quality of life in current clinical practice. The patient's perception of the treatments received is of great help in improving medical procedures, aiming to increase the subject's well-being with no worsening of the quality of the techniques. The use of instruments such as questionnaires enables us to quantify the overall satisfaction and quality of life of the patient, playing a key role in clinical investigation [8]. An example of these tools is the Sucma14 questionnaire, described in 2001 to evaluate the quality of life following outpatient surgeries. It has proven to be a valid and reliable instrument to measure patient satisfaction in the postoperative period of these procedures [9].

It is considered relevant to perform studies to investigate the reliability of submaxillectomies in an outpatient basis, as day surgery has many benefits, the high incidence submandibular gland removal and the lack of the literature of this type of procedures with no hospital stay.

This study aims to establish both the security of the procedure and the patient satisfaction following outpatient submaxillary gland removal.

\section{Methods}

A longitudinal retrospective study was designed enrolling all the patients who underwent a submandibular gland removal with active drain in an outpatient basis between March 2016 and June 2020 in the Oral and Maxillofacial Surgery Department of the University Hospital of Guadalajara, Spain. Inclusion criteria were patients who had a submaxillectomy due to benign disease according to the preoperative assessment. Patients were excluded if their residency was over $100 \mathrm{kms}$ far away from the hospital or had transference problems to the health center, no matter how far away they lived. In addition, patients who required hospital stay because of the anesthetic preoperative assessment were also excluded.

Submandibular gland removal was performed through cervical approach, closing the wound after placing an active drain (10FR Silicone vac drain with $100 \mathrm{cc}$ silicone C.W.V. reservoir (FortuneMed $\left.{ }^{\circledR}\right)$ ).

Postoperative instructions were given to all the patients until removal of the drain, consisting in home rest, avoid sudden movements and transfer to the hospital emergency unit in case of severe pain, sudden and severe amount of drainage or difficult swelling or breathing.

Daily monitoring was made until the removal of the drain. Patients were scheduled for an appointment one week after the surgery for suture removal and a final appointment 1 month after the procedure.
Patient demographic variables were collected including weight, height, body mass index $\left(\mathrm{kg} / \mathrm{m}^{2}\right)$, the physical status of the American Society of Anesthesiologists (ASA) and comorbidities that could potentially have any influence in the immediate postoperative period, such as chronic obstructive pulmonary disease, sleep apnea-hypopnea syndrome, high blood pressure, diabetes or use of anticoagulants or antiplatelet treatments. Surgery variables included side of the surgery, etiology leading to gland removal, amount of drainage in cc in the first three postoperative days (visual scale marking daily debit drainage), total drainage throughout the monitoring, day of drain removal, complications (seroma, hematoma, infection or facial nerve injury) and hospital readmission need because of complications associated with the surgery.

Patients were contacted by phone one month after the surgery to fulfill the SUCMA 14 questionnaire in order to evaluate the patient satisfaction degree with the outpatient surgery. This questionnaire was formulated by García Fernández et al. [9] and includes 14 items with 4 possible answers, ranging from 0 (very satisfied) to 3 (no satisfaction) (range 0-42). These items are divided into three blocks: quality of medical attention satisfaction, major outpatient surgery satisfaction (MOSS) and postoperative monitoring satisfaction. Attention was focused on the second block or MOSS, considering the intimate relationship of its items with the surgery itself (range 0-18). The sample was studied considering two different groups: Demographic and SUCMA 14 data were analyzed accounting the total number of patients, while etiological, surgical and postoperative variables were considered accounting the total of surgical procedures.

Before descriptive analysis was made, missing data analysis was implemented. Variables showing normal distribution were studied using mean and standard deviation, while variables with asymmetric distribution were tested with median and interquartile range. Spearman's coefficient was used to evaluate the possibility of association between time until drain removal and patient satisfaction degree measured with SUCMA14 and MOSS. Data were analyzed using Stata 14.2 (StataCorp, College Station, TX).

\section{Results}

Between March 2016 and June 2020, 15 patients underwent submandibular gland removal accounting for a total of 16 submaxillectomies on an outpatient basis. No missing data were found when the sample size was considered as the number of patients; however, when it was considered as the number of surgical procedures, five missing values were found in both the first and second postoperative day 
drainage measurement variables. Follow-up was complete in the whole sample.

Demographic characteristics are shown in Table 1. $73.3 \%$ (n11) of the subjects were female. Most of the patients were classified as ASA 2 (60\% (n9)), and 53.3\% had comorbidities that could have any influence in the postoperative period.

One patient underwent a bilateral submandibular gland removal, with the rest of the sample following unilateral procedures. Twelve of these surgeries were performed due to inflammatory etiology and the three remaining ones because of neoplasms (two pleomorphic adenomas and one clear cell carcinoma metastasis).

Daily drainage was collected in 11 procedures, with the median of the first postoperative day drainage being $10 \mathrm{cc}$. Active drains were removed on an outpatient basis in the first postoperative day in $56.3 \%$ of the surgeries; only $6.2 \%$ of these drains had to be removed in the third postoperative day.

One patient was referred to the hospital before the drain removal in the second postoperative day but was discharged from the emergency room as no hospital stay was needed.

No complications associated with the outpatient management were recorded. One patient $(7.14 \%)$ had temporary facial nerve palsy with full recovery in the following months.

Low values were found in both SUCMA 14 (Fig. 1) and the six items of MOSS (Fig. 2) in most of the patients. Mean values considering the 15 patient sample were 7.06 and 3.73 , respectively. $86.67 \%$ of the patients would likely or very likely be operated in an outpatient setting once again and considered the hospital stay adequate. $88.67 \%$ of the patients referred little or no pain and $66.67 \%$ were not afraid or concerned about the fact of staying at home after the surgery.

Correlation coefficient was used to explore the possible association between time until drain removal and patient satisfaction degree measured with SUCMA14 and MOSS. Both coefficients were statistically non-significant $(0.03$ ( $p=0.92)$ and $0.06(p=0.84)$, respectively, so the possibility of independence between those variables cannot be ruled out.

\section{Discussion}

Outpatient surgery is used in those procedures that allow early wandering and reduced hospital stay. This fact benefits not only the individuals but also the health system ${ }^{9}$, aiming to reduce cost-per-process with no reduction of quality of healthcare provided or results while being accepted by the patient [3].

The current COVID-19 pandemic background has forced the prioritization of medical resources to deal with the high number of patients suffering from this infection; thus, day surgeries are recommended whenever possible.

Submandibular gland removal is a common surgical procedure in submaxillary gland pathology. Cervical approach is the most frequent technique in this surgery, providing an adequate visualization of the surgical field. Other approaches, such as the intraoral approach, have
Table 1 Sample demographic characteristics

\begin{tabular}{llll}
\hline Demographic variables & $n=15$ & Variables by procedure & $n=16$ \\
\hline Age (years) & $60.2(13.9)^{*}$ & Location & \\
Gender & & Left & $7(43.8 \%)$ \\
Male & $4(26.7 \%)$ & Right & $9(56.2 \%)$ \\
Female & $11(73.3 \%)$ & Etiology & \\
BMI $\left(\mathrm{kg} / \mathrm{m}^{2}\right)$ & $27,1(5.3) * *$ & Oncologic & $3(18.8 \%)$ \\
Smoke consumption & & Sialoadenitis \\
Non-smoker & $8(53.3 \%)$ & Pathology & $13(81.2 \%)$ \\
Ex-smoker & $2(13.3 \%)$ & Oncologic & \\
Active smoker & $5(33.3 \%)$ & Sialoadenitis & $3(18.8 \%)$ \\
Comorbidity & & Complications & $13(81.2 \%)$ \\
Yes & $8(53.3 \%)$ & Yes & $1(6.3 \%)$ \\
No & $7(46.7 \%)$ & No & $14(93.7 \%)$ \\
ASA Score & & Drain removal (days) & $1(1)^{* *}$ \\
I & $5(33.3 \%)$ & Drain removal on first day & $9(56.3 \%)$ \\
II & $9(60.0 \%)$ & Drainage on first day $(c c)$ & $10(5)^{* *}$ \\
III & $1(6.7 \%)$ & & \\
\hline
\end{tabular}

*average (standard deviation) $* *$ median (interquartile range) 
Fig. 1 SUCMA 14 results

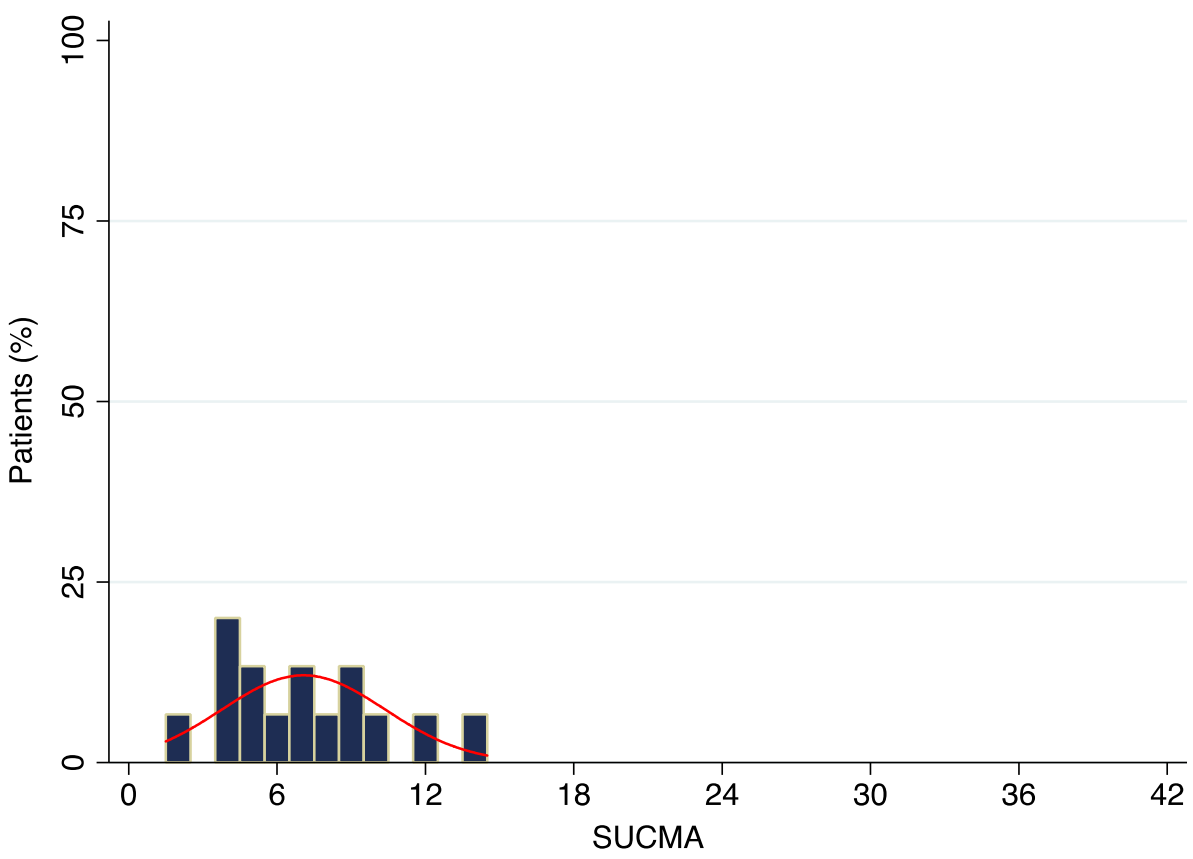

Fig. 2 MOSS results

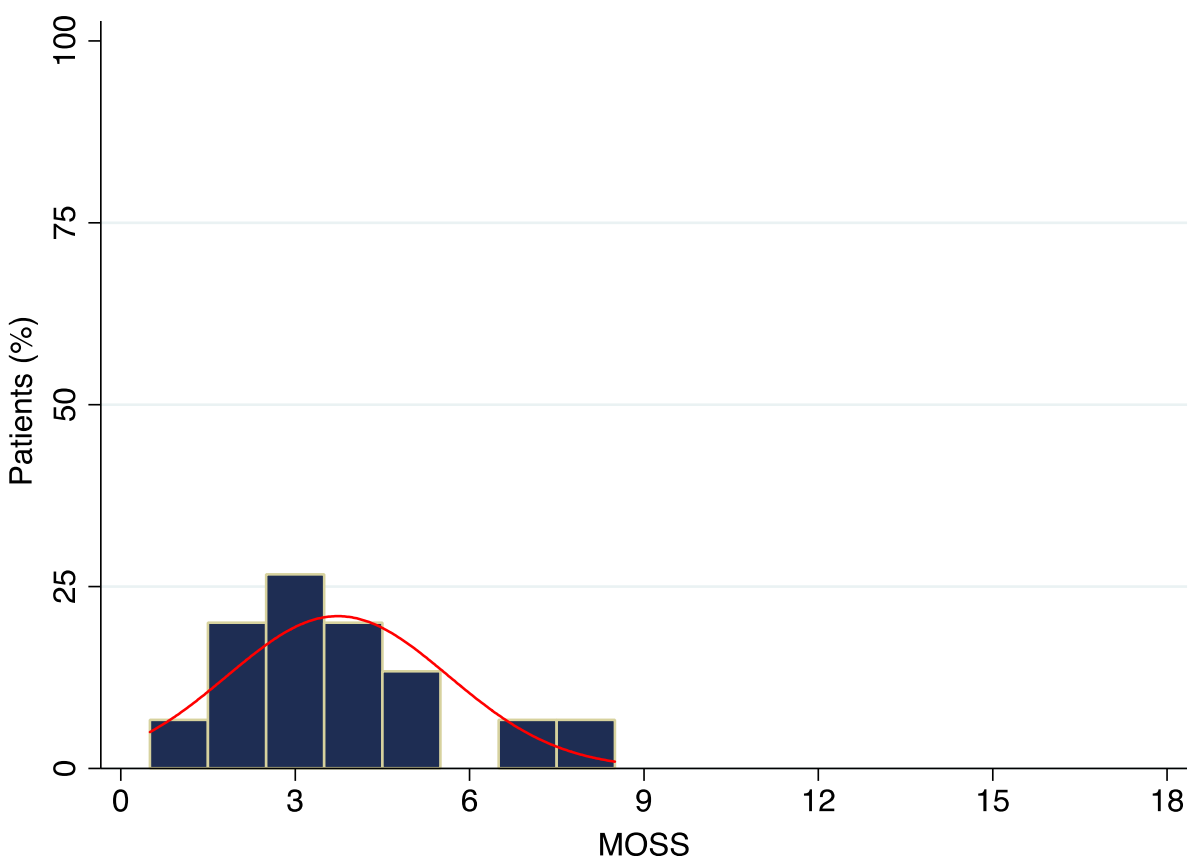

been described but are not so widely used because of the bleeding risk and the possibility of injury of the lingual nerve and the marginal branch of the facial nerve [4]. It has also been described the endoscopic and robotic approach [10]. All submandibular glands were removed through cervical approach in our series.

Although several authors have considered the submaxillectomy without drain a safe procedure, leaving or not hemostatic agents before closure in the surgical field $[7,11-13]$, the mainstream is use active drains $[5,6,14]$; this was the procedure followed in our patients.
Drains in this study were not removed if drainage was over $25 \mathrm{cc}$ or below $25 \mathrm{cc}$ with signs of blood accumulation in the surgical field. Mofle et al. [2] set the drainage limit for drain removal after a parotidectomy in $15 \mathrm{cc} / 8 \mathrm{~h}$. Moreover, they state that drainage after parotid removal is lower in benign neoplasms than malignant tumors [2]. In this study, the drain was removed two days after the surgery in a patient with a clear cell metastasis, slightly higher than our mean removal value (1,47 days). One of the determinant factors for hospital stay in the postoperative period of a submaxillectomy is the removal of the drain 
[11]. Patients stay at least one day even in those institutions with no postoperative active drain [7, 11, 12]. Mean hospital stay after a submandibular gland removal ranges from 2 to 3 days $[11,15]$.

No complications associated with outpatient surgery were recorded, and none of the patients had to be readmitted and stay in hospital. These data confirm that day surgery is a safe procedure after a submaxillectomy. A high percentage of our patients would like to be operated on an outpatient basis, considered an adequate hospital stay and experienced low or no pain after the surgery, confirming the high degree of patient satisfaction after an outpatient submandibular gland removal.

Despite the fact that our data did not show statistical association between time until drain removal and degree of patient satisfaction, it should be taken into account the influence of the small sample size of this study on the possibility of undetecting such relationship.

Although our results are promising, this investigation has some limits such as the retrospective nature of this investigation, the lack of control group and the sample size. This initial exploratory research supports the need for further prospective studies with larger samples that could help to confirm our preliminary results.

Outpatient submandibular gland removal using active drains is a safe procedure, with few or no complications regarding inpatient surgery. In addition, the SUCMA14 questionnaire reveals the high degree of patient satisfaction and acceptance of performing this surgery on an outpatient basis.

Author contributions All authors contributed to the study conception and design. Material preparation, data collection and analysis were performed by JAGM, AAFV and JFAL. The first draft of the manuscript was written by JAGM, and all authors commented on previous versions of the manuscript. All authors read and approved the final manuscript.

\section{Declarations}

Conflict of interest The authors manifest that there are no sources of support in the form of grants and no conflicts of interest or competing interests.

\section{Ethical approval}

Ethical approval was given by the Guadalajara University hospital research ethics committee.

Informed consent All patients were informed about the research and approved to participate in it signing the informed consent.

\section{References}

1. Flach S, Hey SY, Lim A, Maniam P, Li Z, Donnan PT, Manickavasagam J (2020) Outpatient (same-day discharge) versus inpatient parotidectomy: a systematic review and meta-analysis. J Clin Otolaryngol 45:529-537. https://doi.org/10.1111/coa. 13519

2. Mofle PJ, Urquhart AC (2008) Superficial parotidectomy and postoperative drainage. Clin Med Res 6:68-71. https://doi.org/10. 3121/cmr.2008.787

3. Fernández Torres B, García Ortega C, Marquez C, Fontan IM (1999) Caracterización de la cirugía mayor ambulatoria en un hospital general básico. Rev Esp Salud Publica 73:71-80

4. Miloro M, Ord RA, Pazoki AE (2004) Salivary gland disease and tumors. In: Miloro M (ed) Peterson's principles of oral and maxillofacial surgery. BC Decker Inc, London, p 675

5. Diseases of the salivary glands In: Anil MN, (ed) Textbook of oral and maxillofacial surgery. New Delhi: Jaypee Brothers Medical Publishers (P) Ltd, (2016), p. 776

6. Dias FL, Lima RA, Cernea CR (2007) Management of tumors of the submandibular and sublingual glands. In: Myers EN, Ferris RL (eds) Salivary gland disorders. Springer, Berlin, p 358

7. Bannister M, Ah-See K (2014) Safety of the haemostatic agent Surgiflo ${ }^{\circledR}$ in excisions of the submandibular gland: our experience in 17 cases. Br J Oral Maxillofac Surg 52:e134-e135. https://doi.org/10.1016/j.bjoms.2014.05.014

8. Wax MK, Talmi YP (2016) Quality of life after salivary gland surgery. Adv Otorhinolaryngol 78:189-197. https://doi.org/10. $1159 / 000442140$

9. García Fernández FP, Pancorbo Hidalgo PL, Rodríguez Torres MC, Rodríguez Torres MA, Alcázar Iglesias M, Pereira BF (2001) Construcción y validación de un cuestionario para valorar la satisfacción de los usuarios de cirugía mayor ambulatoria. Enferm Clin 11:146-154

10. Yang TL (2018) Robotic surgery for submandibular gland resection through a trans-hairline approach: the first human series and comparison with applicable approaches. Head Neck 40:793-800. https://doi.org/10.1002/hed.25058

11. Şahin B, Esen E, Başaran B (2020) Drainless resection of the submandibular gland with facial vessel preservation: a comparative study. J Stomatol Oral Maxillofac Surg 121:501-505. https://doi.org/10.1016/j.jormas.2019.12.017

12. Park HS, Lee SM, Lee KH, Chun MS, Kim HS (2020) Safety of drainless excision of the submandibular gland. Braz J Otorhinolaryngol 86:626-631. https://doi.org/10.1016/j.bjorl.2019.04.010

13. Hussain A, Murray DP (2004) Modified submandibular sialoadenectomy. Ear Nose Throat J 83:770-771

14. Andersen PE (2011) Submandibular gland excision. In: Cohen JI, Clayman GL (eds) Atlas of head and neck surgery. Elsevier Saunders, Philadelphia, pp 240-241

15. Chiesa C, Valldeperes A, González J, Larruscain E, Sistiaga J, Altuna X (2019) Neurological complications and quality of life after submandibular gland resection. A Prospective, non-randomized, single-centre study. Otolaryngol Pol 73:32-37. https:// doi.org/10.5604/01.3001.0013.4120

Publisher's Note Springer Nature remains neutral with regard to jurisdictional claims in published maps and institutional affiliations. 